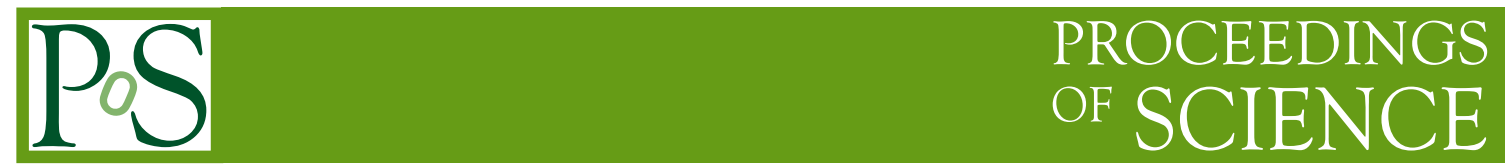

\title{
Search for Sterile Neutrinos at RENO
}

\author{
Jiwoong SEO* \\ Department of Physics, Sungkyunkwan University, Suwon, Korea \\ E-mail: Gwseo115egmail.com
}

The RENO experiment has successfully measured $\theta_{13}$ using the disappearance of electron antineutrinos in three-flavor neutrino oscillations. We search for sterile neutrinos in four-flavor oscillation model using roughly 1500 days of data collected by the RENO experiment. We have not seen any positive signal and obtain an excluded region of the oscillation parameters. We present an excluded contour plot in $\sin ^{2} 2 \theta_{14}-\Delta m_{41}^{2}$ space.

The 39th International Conference on High Energy Physics (ICHEP2018)

4-11 July, 2018

Seoul, Korea

${ }^{*}$ Speaker. 


\section{Introduction}

The oscillation parameters are determined using the far to near ratio of neutrino energy spectra measured by the RENO experiment. Observed spectra are compared with Monte Carlo spectra generated in four-flavor neutrino frame. The oscillation parameters $\sin ^{2} 2 \theta_{14}$ and $\Delta m_{41}^{2}$ are set as free variables while the other oscillation parameters are constrained to the RENO measurement values or PDG values. In order to obtain an excluded region in the parameter space, we use the $\chi^{2}$ fitting based on the pull method[四].

\section{Result}

By analyzing 2200 days of data taken by the RENO experiment, we search for sterile neutrinos and set an exclusion contour curve as shown in figure 1(a). Observed spectra are also compared to sterile neutrino predictions in figure 1(b).

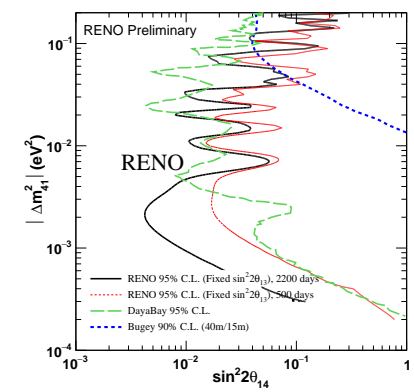

(a)

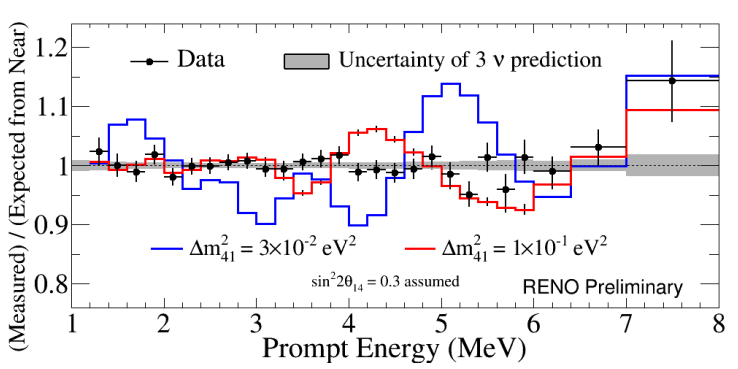

(b)

Figure 1: (a) Exclusion contour curve in $\sin ^{2} 2 \theta_{14}-\Delta m_{41}^{2}$ plane. The black(red) solid curve represents the $95 \%$ C.L. exclusion contour curve using 2200(500) days of RENO data. For comparison, we show the results from Bugey[[]] and Daya Bay experiments[B]. (b) Ratio of observed energy spectrum to prediction at far detector. The grey band represents the uncertainty of the three-neutrino-flavor oscillation prediction.

\section{Conclusion}

We obtained an excluded region for sterile neutrino oscillation parameters in the $\sin ^{2} 2 \theta_{14}-$ $\Delta m_{41}^{2}$ plane. The RENO experiment is apparently more sensitive in the small $\Delta m_{41}^{2}$ region due to longer baselines from farthest nuclear reactors to the detectors.

\section{References}

[1] S.H.Seo et al. (RENO Collaboration), Spectral measurement of the electron antineutrino oscillation amplitude and frequency using 500 live days of RENO data, Phys. Rev. D 98, 012002 (2018)

[2] Y.Declais et al. (Bugey Collaboration), Search for neutrino oscillations at 15, 40 and 95 meters from a nuclear power reactor at Bugey, Nucl. Phys. B 434503 (1995)

[3] F.P.An et al. (Daya Bay Collaboration), Improved Search for a Light Sterile Neutrino with the Full Configuration of the Daya Bay Experiment, Phys. Rev. Lett. 117, 151802 (2016) 